Archives

$30 \mid 2002$

Foccart - Entre France et Afrique

\title{
"La politique est au bout du réseau ». Questions sur la méthode Foccart
}

Jean-François Médard

\section{(2) OpenEdition}

\section{Journals}

\section{Édition électronique}

URL : http://journals.openedition.org/ccrh/612

DOI : $10.4000 /$ ccrh.612

ISSN : $1760-7906$

Éditeur

Centre de recherches historiques - EHESS

Édition imprimée

Date de publication : 30 octobre 2002

ISSN : 0990-9141

Référence électronique

Jean-François Médard, " «La politique est au bout du réseau ». Questions sur la méthode Foccart », Les Cahiers du Centre de Recherches Historiques [En ligne], 30 | 2002, mis en ligne le 22 novembre 2008, consulté le 10 décembre 2020. URL : http://journals.openedition.org/ccrh/612 ; DOI : https://doi.org/ $10.4000 /$ ccrh. 612

Ce document a été généré automatiquement le 10 décembre 2020.

Article L.111-1 du Code de la propriété intellectuelle. 


\title{
« La politique est au bout du réseau ». Questions sur la méthode Foccart $^{1}$
}

\author{
Jean-François Médard
}

Écrire sur Jacques Foccart constitue pour le politologue un exercice scientifique redoutable dans la mesure où le personnage, en raison du secret dont il s'entourait, de l'influence et de la puissance qu'on lui prêtait, a pris les dimensions d'un véritable mythe. Mais Jacques Foccart n'est pas qu'un mythe. Il a rempli des fonctions importantes auprès du général de Gaulle pendant vingt-trois ans, et a exercé les fonctions officielles de secrétaire général à la Communauté, puis aux Affaires africaines et malgaches pendant quatorze ans, de 1960 à 1969 auprès du général de Gaulle, puis auprès de Georges Pompidou jusqu'en 1974. Certes, tout ce qu'on a pu écrire sur Jacques Foccart ne doit pas être pris pour argent comptant: $s$ 'il a plus que probablement trempé dans des complots et des coups d'État, pensons aux interventions au Nigeria, en Guinée ou au Bénin, il serait ridicule de croire qu'il était derrière chaque coup d'État en Afrique. Comment, dans ces conditions, démêler le vrai du faux? Ou plus modestement comment distinguer ce qui relève du prouvé, du probable, du possible et du faux, a fortiori lorsqu'on ne dispose pas soi-même d'informations de première main?

2 En tout état de cause, s'efforcer d'évaluer les méthodes et l'influence de Jacques Foccart est un exercice indispensable pour éclairer certains des ressorts longtemps cachés et bien cachés de la $V^{e}$ République et des relations franco-africaines. L'ambition de cette note, qui ne constitue qu'une ébauche, n'est pas d'apporter de nouvelles révélations mais seulement de tenter de faire le point, d'une façon plus analytique que factuelle, sur ce que l'on désigne couramment comme le (ou les) réseau(x) Foccart. Cette notion de réseau est en effet au cœur de ce qu'on peut appeler la méthode Foccart, comme elle est au fondement même de ce qu'on désigne maintenant sous le nom de Françafrique, qu'il a, avec le général de Gaulle, contribué à fonder et à orienter. Notre objectif n'est pas, dans ce cadre ci, d'en dénoncer les méthodes, c'est au lecteur d'apprécier, mais d'essayer d'en démonter dans la mesure du possible les mécanismes dans l'état actuel des connaissances. 
Le secret de l'influence de Jacques Foccart, et ce qui démultiplie l'efficacité de son réseau, c'est ce qu'on peut appeler la multipositionnalité de Jacques Foccart, c'est-à-dire le fait qu'il occupe à l'Élysée, dans l'appareil de l'État et au sein du mouvement gaulliste, un certain nombre de positions stratégiques à cheval sur le formel et l'informel. Le réseau de Jacques Foccart s'appuie sur des positions institutionnelles multiples dont il peut mobiliser les ressources pour étendre son influence au sein d'autres institutions et d'autres réseaux : c'est ce que nous appellerons la «toile » de Foccart. En deuxième lieu, on parle couramment, soit du réseau Foccart au singulier, soit des réseaux Foccart au pluriel. Lorsqu'on parle du réseau Foccart au singulier, il s'agit du réseau des réseaux, c'est-à-dire l'ensemble des réseaux de Jacques Foccart au sens large et polysémique du terme, ce qui inclut à un pôle, les réseaux purement relationnels et licites de Jacques Foccart, et à l'autre les réseaux sinon illégaux du moins occultes, les frontières du licite et de l'illicite étant des plus floues. Lorsqu'on évoque les réseaux Foccart au pluriel, on fait plutôt allusion aux réseaux occultes. Il faut donc clairement distinguer deux volets du personnage correspondant à deux types de réseaux, qui se complètent sans s'opposer, le côté purement relationnel, interpersonnel et même amical du personnage, et le volet occulte et éventuellement illicite. Mais il faut auparavant évoquer le rôle de Jacques Foccart dans la Françafrique.

\section{Jacques Foccart et la françafrique}

Parmi les anciennes puissances coloniales, la France est la seule à avoir véritablement cherché et réussi à la fois à maintenir son influence dans ses anciennes possessions bien au-delà des indépendances. Ce système de relations entre la France et la plupart de ses anciennes colonies d'Afrique a pris récemment le nom de Françafrique, au moment même où il traversait une crise sérieuse. Le terme emprunté à Félix Houphouët-Boigny a été détourné de son sens premier en lui donnant une connotation polémique et péjorative par François-Xavier Verschave ${ }^{2}$. Ce n'est pas une raison parce qu'il a pris cette connotation qu'il faut le disqualifier, car il peut être utilisé d'une façon plus neutre et plus analytique pour désigner et nommer ce système de relations tout à fait spécifique que constituent les relations franco-africaines, qui n'a aucun équivalent, sauf peut-être avec les États-Unis et certains pays d'Amérique Latine. Ce système a débouché sur la constitution d'un véritable ensemble franco-africain de nature largement informelle ${ }^{3}$, qui a perduré jusqu'à nos jours et qui n'en finit pas de disparaître. Au plan formel, la Françafrique se caractérise par un certain nombre d'institutions, comme le ministère de la Coopération, aujourd'hui absorbé par le ministère des Affaires étrangères, la zone franc, la francophonie, les accords de coopération civils et militaires et la présence militaire française en Afrique... Sur le plan informel, elle repose sur une infrastructure de réseaux de nature très variée, souvent occultes et illicites.

5 Jacques Foccart peut être considéré, avec le général de Gaulle, comme le père fondateur de la Françafrique, mais c'est ce dernier qui en a été l'inspirateur. Jacques Foccart en a été le maître d'œuvre, et il n'a fait qu'appliquer avec une grande efficacité la stratégie élaborée par le général de Gaulle, ce qui est déjà beaucoup. Il a, pendant quatorze ans, été le véritable fondé de pouvoir du général de Gaulle et plus tard celui de Georges Pompidou pour l'Afrique, manifestant à l'un comme à l'autre une totale fidélité. On ne peut donc pas les opposer. Mais il existait entre eux une sorte de division des tâches qui correspondait grosso modo à la distinction entre niveau stratégique et niveau tactique, en définitive entre 
haute politique et basse politique, et aussi entre politique officielle et publique et politique réelle. Au général de Gaulle, les lignes directrices et les beaux discours à connotation éventuellement tiers-mondiste, comme le discours de Pnom Penh, à Jacques Foccart la gestion quotidienne. La main droite de Charles de Gaulle pouvait ainsi paraître ignorer ce que faisait sa main gauche.

Il y a donc eu, à l'époque, une véritable politique africaine de la France, et pas seulement un certain nombre d'actions publiques. On peut ne pas en partager les objectifs et encore moins les moyens, ce qui est notre cas, mais il faut reconnaître qu'elle a réussi, au sens où elle a été effectivement mise en œuvre. Paradoxalement, l'objectif de la décolonisation menée à bien par le général de Gaulle n'était pas véritablement de quitter l'Afrique, mais de partir pour mieux y rester. En d'autres termes, il fallait que «tout change pour que tout reste la même chose ». Au-delà de la magie du verbe gaullien, tel qu'il s'était exprimé pour la première fois à Brazzaville, il fallait, de façon pragmatique et intelligente, s'adapter au vent de l'histoire, par une stratégie de modernisation conservatrice, permettant de conserver le maximum de positions sur le continent au moindre coût économique et humain. C'est pourquoi, au moment même où l'on octroyait l'indépendance aux anciennes colonies d'Afrique, on la vidait en partie de son contenu.

7 Il ne faut pas perdre de vue que la décolonisation, poursuivie par le général de Gaulle en Afrique noire, a été imposée par lui-même, d'abord contre son propre camp. Il faut garder à l'esprit que les gaullistes du RPF se recrutaient parmi les partisans les plus extrêmes du colonialisme et que Jacques Foccart était le premier d'entre eux. Dès les débuts du RPF, Jacques Foccart, s'est vu assigner la tâche d'implanter le parti outre-mer. Élu conseiller de l'Union française, il a, à l'époque, défendu les positions les plus conservatrices, notamment dans la Lettre de l'Union, qui exprimait très clairement les convictions gaullistes colonialistes de l'époque. C'est de Gaulle lui-même qui a dû faire l'éducation de ses propres partisans récalcitrants.

8 Cet objectif du maintien de la présence française en Afrique, et d'une présence de cette nature, était partie intégrante de la grande politique gaullienne. Il correspondait à une certaine vision stratégique des intérêts de la France visant à assurer les bases de l'indépendance nationale. Cela impliquait, outre la sortie de l'Otan, la possession de l'arme atomique et le déploiement d'une force de frappe s'appuyant sur une industrie nationale de la défense. Elle impliquait aussi le contrôle de matières premières stratégiques comme l'uranium et le pétrole. Il s'agissait d'autre part, au nom d'une certaine image de la grandeur de la France, et d'une vision messianique de son rang dans le monde, partagée d'ailleurs par l'ensemble de la classe politique ${ }^{4}$, de réunir, sous l'aile protectrice de notre pays, une clientèle d'États africains en contrepartie d'une fidélité politique et de certains services rendus.

9 Va s'établir ainsi un système de clientélisme international fondé sur un échange de services entre partenaires inégaux. D'un côté l'État-patron, l'État français, joue un rôle critique de protection militaire et économique pour la survie des dirigeants amis, de l'autre, les États-clients africains témoignent en retour leur fidélité, chaque client africain dépendant plus de la France que la France de chaque client. C'est sur cette base d'intérêts bien compris que s'est fondée et a prospéré la Françafrique. Il faut bien comprendre que, dans l'échange de type clientéliste, le client a davantage besoin du patron que le patron $\mathrm{du}$ client. Le besoin du client est critique. C'est sur cette situation asymétrique que se fonde la dépendance du client. L'intérêt du recours à une interprétation en termes de clientélisme international, par rapport aux théories de la dépendance, c'est qu'outre leur 
économisme simpliste ces dernières ne considèrent les acteurs africains comme de simples marionnettes. L'échange, même entre inégaux, repose sur le pouvoir de marchandage respectif des acteurs, donc sur une certaine latitude d'action des acteurs les moins puissants. Lorsqu'on observe le comportement de personnages comme Félix Houphouët-Boigny ou Omar Bongo, il est évident qu'on est en face d'acteurs politiques à part entière, au point qu'on a pu se demander parfois qui était la marionnette de qui. Mais le problème que pose le recours au modèle clientéliste, c'est que la relation de clientèle est une relation entre des personnes et non pas entre les abstractions que sont les États. Mais justement, dans le cas franco-africain, les relations entre l'État français et les États africains francophones ne sont pas des relations comme les autres. Les relations entre les États sont médiatisées par des relations personnelles, ou inversement, les relations entre les personnes, les échanges sociaux, sont instrumentalisés par ces personnes au profit (mais parfois aux dépens) des États. Le clientélisme françafricain s'enracine en effet dans tout un tissu de relations d'amitié, d'ordre affectif, entre les élites africaines et les élites françaises qui a souvent été souligné, et qui est essentiel pour comprendre la Françafrique. C'est ce qui a donné à cette dernière cette «dimension charnelle ", que soulignent certains auteurs, et qui a nourri tous les discours en termes de parenté sur la "grande famille franco-africaine $"^{5}$. Mais de la grande politique, on glisse facilement à la basse politique, du clientélisme franco-africain on est passé à une véritable patrimonialisation des relations franco-africaines, et à une certaine privatisation de ces relations franco-africaines, en mêlant allégrement échanges sociaux, échanges économiques et échanges politiques et éventuellement le recours à la force ${ }^{6}$. Jacques Foccart a été l'homme-orchestre de cette politique. Pierre Biarnès, cité par Philippe Gaillard, a résumé ainsi les constantes de la politique de la France à l'égard de ses anciennes colonies qu'il a été chargé d'appliquer :

Consolider le pouvoir des dirigeants qui jouaient loyalement le jeu de l'amitié franco-africaine... et faire sentir le mors à ceux qui regardaient un peu trop dans d'autres directions ; contrer en même temps les visées des puissances concurrentes dès qu'elles étaient jugées menaçantes ${ }^{7}$

À cette citation, Jacques Foccart répond :

Il faut bien dire que c'est un peu vrai. J'ajoute que nous avons orienté, finalement, plus qu'on ne croit. Essentiellement par des contacts directs, nous avions de l'influence sur l'évolution des uns et des autres ${ }^{8}$.

10 Il ne faut cependant pas avoir une vision trop irénique de cette pratique du clientélisme. Le clientélisme repose sur un échange de faveurs. Or qui dit faveurs, dit défaveurs, qui dit favoritisme dit son contraire, "faire sentir le mors ", c'est-à-dire sanctionner ceux qui ne vont pas dans la bonne direction. Traduit en termes concrets, cela veut dire se débarrasser, politiquement ou même physiquement, de ceux qui ne marchent pas dans la bonne direction. C'est Jacques Foccart qui a su, à son niveau, orchestrer ce clientélisme franco-africain, en jouant de la faveur et de la défaveur, celle-ci prenant parfois un caractère musclé. Un mot revient toujours lorsqu'on évoque Jacques Foccart et la Françafrique, c'est celui de réseau. Ainsi Stephen Smith et Antoine Glaser nous disent qu'en Afrique «la politique est au bout du réseau ». Sous le règne de Jacques Foccart, on peut dire qu'il y a adéquation entre les réseaux Foccart et la Françafrique. Ce sont les réseaux Foccart qui irriguent et animent la Françafrique. Après Foccart, il y aura toujours des réseaux, mais ils seront multiples, tantôt concurrents et tantôt alliés. Si l'on veut donc caractériser la méthode Foccart, il faut recourir à la notion de réseau. Toute cette politique s'appuyait donc sur le fameux réseau Foccart dont l'infrastructure formelle, ou 
plutôt l'interface entre le formel et l'informel, constitue ce que nous appelons la « toile de Jacques Foccart» (au sens de « web»).

\section{La « toile » de Jacques Foccart}

11 Le réseau Foccart, en tant que réseau relationnel, est certes informel, mais il s'articule à des structures publiques et privées et aux acteurs qui fonctionnent à l'intérieur de ces structures. Derrière les réseaux, il y a souvent des structures. Inversement, ces réseaux informels constituent comme l'ombre portée des structures. Le secret de l'influence de Jacques Foccart, c'est la nature de son réseau et de son articulation à des structures politiques et économiques, publiques et privées. C'est ce qui lui a permis d'être informé et d'agir à partir de canaux diversifiés, formels et informels, privés et publics, licites et illicites. Foccart était moins au sommet d'une pyramide de réseaux, qu'au centre d'une toile, web, dont il était le point convergent, le «nexus» (Daniel Bach). Au faite de sa puissance, particulièrement sous le général de Gaulle, le réseau Foccart peut s'appuyer sur des supports formels diversifiés et produisant entre eux un effet de synergie. En même temps, ses réseaux informels pourront exercer leur influence non seulement directement au cœur de ces supports institutionnels, mais en même temps indirectement au sein d'institutions où il pourra faire sentir son autorité, sans pour autant y détenir des fonctions officielles. Par la suite, et progressivement avec Georges Pompidou, il perd le contrôle formel de certains de ses supports et les ressources qui y sont attachées. Son réseau va commencer à s'éroder. Il va en même temps être concurrencé par d'autres réseaux, comme celui de Charles Pasqua, un dissident du SAC. La rupture décisive apparaît lorsqu'il est renvoyé par le président Valéry Giscard d'Estaing nouvellement élu. Jamais il ne retrouvera la même influence, car dépourvu de toute position officielle, ses réseaux privés ne pourront plus s'appuyer directement sur ces structures. Mais d'un autre côté, il sera influent grâce à ses réseaux relationnels privés; il s'efforcera de les entretenir à coups de téléphone et de visites réciproques, et il continuera jusqu'à sa mort d'exercer un contrôle au sein d'une Françafrique de plus en plus éclatée. Il reprendra même officiellement du service auprès de Jacques Chirac. Mais les réseaux, comme les hommes, vieillissent. Les supports institutionnels de Jacques Foccart furent de trois ordres : ils recouvrent ses rapports avec les présidents qu'il a successivement servis, ses rapports avec l'appareil de l'État et ses rapports avec le mouvement gaulliste. Il est là encore difficile de départager dans ces relations ce qui relève de l'officiel et de l'officieux.

\section{La relation de proximité avec les présidents}

12 Le premier support institutionnel, c'est évidemment l'Élysée et la première ressource de Jacques Foccart est sa proximité à la fois institutionnelle et personnelle avec les présidents de Gaulle et Pompidou, eux-mêmes placés par la Constitution de la $\mathrm{V}^{\mathrm{e}}$ République au sommet de l'État. Cela est démultiplié, pour l'Afrique, par le fait qu'elle relève directement du domaine réservé et donc de la compétence du président, à l'exclusion du Premier ministre et du Parlement. Mais tout dépend des relations de confiance qu'il entretient avec le président. Il est pendant dix ans le plus proche collaborateur du général de Gaulle. Cette proximité correspond au cumul de plusieurs responsabilités d'importance stratégique, qu'il exerce auprès du Général et au sein du mouvement gaulliste, dont il est la tête. Foccart n'a pas cherché à faire une carrière 
politique ni à devenir ministre. Il a choisi le rôle du conseiller de l'ombre, tout en exerçant des fonctions tout à fait officielles. Mais on a longtemps ignoré quelles étaient ses attributions précises en tant que conseiller. Il y a toujours eu une sorte de flou dans ce domaine, d'autant plus que la démarcation entre le conseiller public et le conseiller privé du Général est difficile à faire.

13 La clé de l'influence de Jacques Foccart et de sa puissance repose d'abord sur cette relation privilégiée qu'il a su établir avec les deux premiers présidents de la $\mathrm{V}^{\mathrm{e}}$, et d'abord avec le premier d'entre eux, Charles de Gaulle. C'est cela qui a conditionné tout le reste. Ancien des réseaux de la France libre, Jacques Foccart n'a rencontré personnellement le Général - pour la première fois - qu'en décembre 1946. Très vite, en 1947, ce dernier lui assigne des missions d'implantation du nouveau parti, le Rassemblement du peuple français, dans l'Ouest où il avait mené ses activités de résistance, dans le Nord, puis dans les Antilles dont il était originaire, et enfin dans l'ensemble des départements et territoires d'outre-mer dont l'Afrique. Ainsi, dès 1947, Foccart s'occupe des affaires africaines du RPF. Il devient le trésorier général du Rassemblement et le secrétaire général de l'Upang, association en charge de collecter des fonds. En novembre 1953, il est nommé secrétaire adjoint du RPF, plus spécialement chargé des territoires d'outre-mer, puis secrétaire général en 1954. Il voit alors le général de Gaulle toutes les semaines, l'accompagne dans ses trois grands voyages outre-mer, où sa connaissance des élus s'avère très précieuse. Il devient ainsi le Monsieur Afrique, l'intermédiaire obligatoire entre le général de Gaulle et ses interlocuteurs africains, ainsi qu'entre le Général et les ministres en charge des questions africaines. Le Général lui fait une entière confiance et se repose sur son jugement. En retour, la loyauté de Jacques Foccart à son égard paraitt sans faille. S'instaure alors une division du travail fort efficace. Charles de Gaulle était informé des affaires importantes et les décisions étaient prises avec son accord. En ce qui concernait les affaires secondaires, il n'était pas tenu au courant du détail des préparatifs ${ }^{9}$ :

Le Général était plus flou sur les détails et il me laissait, finalement, une assez grande marge d'interprétation sur le choix des moyens ${ }^{10}$.

On peut penser qu'il y avait beaucoup de non-dit dans cette relation. À partir du moment où il $\mathrm{y}$ avait accord sur les objectifs et confiance du général de Gaulle dans la loyauté de son collaborateur, ce dernier se fiait à Jacques Foccart pour décider ce qui relevait de l'important et du secondaire. Non seulement le Général n'avait pas, à son niveau, à entrer dans les détails, mais il n'y avait pas intérêt. Il y a certains « détails » dont il vaut mieux que le président ne s'occupe pas directement, le collaborateur prenant alors ses responsabilités à ses risques et périls. On entre ici dans le domaine de la raison d'État, que Jacques Foccart invoque à plusieurs reprises pour justifier son silence. Jacques Foccart reconnait l'existence de ce non-dit :

Je souligne-parce que cela restera une constante dans mes relations avec le Général - qu'il y a eu à cette époque, comme il y aura en Afrique, des choses non dites entre nous, concernant mon action et mes initiatives. Sauf parfois, au détour d'une conversation, une allusion: "Oui, Foccart, je sais très bien que... ». Il se doutait plus ou moins de ceci ou de cela dont je ne lui parlais pas, mais il ne posait pas de questions. Il contrôlait l'essentiel et beaucoup de détails, sans vouloir, pour autant, connaître certains faits précis qui auraient appelé des explications compliquées et peut-être déplaisantes ${ }^{11}$.

Rien ne permet d'affirmer que Jacques Foccart ait abusé de cette confiance en manipulant le général de Gaulle. On n'a pas l'impression à propos de l'affaire Ben Barka que le 
Général ait soupçonné Jacques Foccart d'avoir agi dans son dos ni que sa colère était feinte : le mystère demeure. Cela ne veut pas dire que Jacques Foccart n'ait pas cherché à l'influencer, ce qui après tout est bien le rôle d'un conseiller. Par exemple, selon Daniel Bach, il semble bien que la décision d'aider le Biafra contre le Nigeria ait été prise par de Gaulle sur les conseils à la fois d'Houphouët-Boigny et de Jacques Foccart ${ }^{12}$.

La relation avec Georges Pompidou a été, elle aussi, empreinte de confiance, mais elle ne se situait pas sur le même plan. Ils étaient amis de longue date, et Jacques Foccart qui bénéficiait de l'aura de sa proximité avec le général, avait soutenu Pompidou sans équivoque contre les défenseurs du gaullisme pur et dur. Pour des raisons d'ouverture au centre, imposée par les contraintes électorales, Georges Pompidou avait tergiversé avant de rétablir Foccart dans ses fonctions, après son renvoi par Poher. Mais il ne l'a pas rétabli dans la plénitude de ses anciennes fonctions sauf pour l'Afrique et les Territoires et départements d'outre-mer (TOM-DOM). Jacques Foccart ne le rencontrait qu'une fois par semaine et non pas quotidiennement. D'ailleurs, Georges Pompidou était fortement influencé par le tandem Juillet-Garraud. Mais en ce qui concernait l'Afrique, Jacques Foccart restait le fondé de pouvoir de Georges Pompidou. Le président s'appuyait très largement sur lui, Jacques Foccart étant considéré comme le plus compétent, celui qui « connaissait les Africains» et qui entretenait avec eux les relations les plus étroites. La politique africaine de Georges Pompidou se situait dans la continuité de celle du Général, mais avec une inflexion plus forte vers le réalisme économique.

La rupture fondamentale va intervenir avec l'élection de Valéry Giscard d'Estaing qui renvoie Jacques Foccart et prend beaucoup plus directement en main la politique africaine de la France. Le secrétariat général aux Affaires africaines et malgaches est réduit à la portion congrue. La politique du nouveau président va évoluer dans le sens d'une certaine continentalisation, une accentuation de la dimension économique, mais aussi un interventionnisme militaire plus poussé. Jacques Foccart est alors sur la touche, mais il est toujours présent dans l'ombre et cultive ses réseaux. Il va progressivement reprendre du service avec Jacques Chirac.

Les rapports avec Jacques Chirac sont plutôt tièdes au début, Jacques Foccart n'appréciant guère certaines de ses initiatives. Mais il devient son conseiller Afrique à la mairie de Paris et le suivra quand il sera Premier ministre puis président de la République. Une relation très confiante et amicale s'établira une fois de plus, et c'est lui qui rétablira les ponts entre Chirac et Mobutu. Mais il était alors âgé et malade. Le moins qu'on puisse dire, c'est qu'il était alors dépassé par les événements et n'avait pas pris conscience des mutations que traversait l'Afrique.

\section{Les relations avec l'appareil de l'état}

Dans l'organigramme, Jacques Foccart est secrétaire général de la Communauté ; avant de devenir secrétaire général aux Affaires africaines et malgaches. Il dirige officiellement une administration en charge de l'Afrique qui, issue de l'éphémère Communauté, va se prolonger jusqu'à aujourd'hui sous le nom de cellule africaine de l'Élysée. Sans avoir le rang de ministre, encore que souvent le Général lui attribue une place protocolaire supérieure à celle des ministres, il est une sorte de super ministre de l'Afrique à l'Élysée. Il chapeaute ainsi, à son niveau, les administrations qui ont à faire avec l'Afrique francophone, qu'il s'agisse du ministère de la Coopération, du ministère des Affaires étrangères ou de celui de la Défense nationale. Tout en étant rattaché ainsi à la 
présidence, il n'a de compte à rendre à personne d'autre qu'au président. Au début, son administration disposait de moyens importants. Ses services étaient logés à l'hôtel de Noirmoutier et il disposait d'un personnel d'une centaine de personnes. Par la suite, ces moyens vont progressivement se réduire.

Mais il est aussi conseiller du président. En tant que conseiller, il est en charge des départements d'outre-mer qu'il suit d'aussi près, et avec la même compétence et connaissance du terrain, que les pays africains. Il intervient ainsi dans toutes les nominations de diplomates et de fonctionnaires concernant les TOM-DOM et l'Afrique francophone. Surtout, en tant que conseiller du Général, il est chargé de superviser au niveau de l'Élysée tout ce qui concerne le renseignement et la sécurité. Il coiffe donc les services de renseignement, le SDECE qui dépend au début du Premier ministre et plus tard, après l'affaire Ben Barka, de la Défense nationale, la DST et les Renseignements généraux qui dépendent du ministère de l'Intérieur. Il entre ainsi en conflit avec Constantin Melnik? qui supervise le SDECE auprès de Michel Debré, Premier ministre, mais, nous dit-il, « en réalité, c'est l'Élysée qui tranche ${ }^{13}$. Il affirme cependant n'avoir pas été impliqué en Algérie ni dans la lutte anti-OAS, qui ne relevait pas de ses compétences. Les relations avec l'État sont difficilement séparables de ses relations avec le mouvement gaulliste.

\section{Les relations au mouvement gaulliste}

21 En tant que conseiller privé du général, il est en charge du mouvement gaulliste. Cela inclut officiellement le RPF, dont il est secrétaire général adjoint depuis 1953 et secrétaire général depuis 1954. Il n'est pas en charge directement de l'UNR ni des partis qui lui succéderont, mais il est l'un de ses plus influents barons qui se réunissent périodiquement à déjeuner. Il est chargé par le général de Gaulle de suivre les questions électorales, ce qu'il fait de très près. Il semble aussi, qu'en liaison avec ses responsabilités africaines, il joue un rôle dans le financement du parti ${ }^{14}$. Enfin, il est responsable, officieusement sinon officiellement, du Service d'action civique (SAC). Cette organisation, créée en 1959, avait pris la suite du service d'ordre du RPF de Ponchardier. Elle recrutait au début parmi des anciens de la Résistance. Le recrutement s'est par la suite élargi à des milieux plus populaires et davantage portés à l'action directe et à l'activisme. Idéologiquement, le SAC se situait à l'extrême droite du gaullisme. Il recrutait fortement chez les policiers et aussi chez les truands, ce qui explique l'existence des fameux «barbouzes », et que le SAC ait périodiquement défrayé la chronique judiciaire jusqu'à sa dissolution par le gouvernement socialiste à la suite du massacre d'Auriol. Jacques Foccart n'a reconnu explicitement qu'il en était le patron que récemment. Il relate dans son journal les réunions du SAC, sans entrer beaucoup dans les détails. Il éprouve à l'égard de ses membres une sorte de sympathie un peu condescendante (ce sont de braves gens), et partage leur sensibilité idéologique, tout en s'efforçant souvent de les calmer. À la question: "Faites-vous partie de la hiérarchie du Service d'action civique?», il répond:

Officiellement non, mais j'en suis le patron jusque-là incontesté. La plupart des bruits qui ont couru et qui courent encore sur mes activités sont faux. Mon autorité sur le SAC, elle, est bien réelle, bien que je ne l'aie pas reconnue publiquement. Mais elle va s'affaiblir avec la relève des générations... ${ }^{15}$. 

clandestine et de la résistance contre l'occupant nazi et le régime de Vichy. Il trouve son origine organisationnelle dans les réseaux de résistance et d'action clandestine, qui dépendaient des services secrets gaullistes, notamment le BCRA à Londres, puis à Alger. Les réseaux de résistance et les services secrets gaullistes sont la matrice des réseaux gaullistes de l'après-guerre. Le SDECE, service officiel de contre-espionnage, est lui-même issu des divers services qui se sont succédé et qui, à l'origine, étaient des réseaux gaullistes. Même si par la suite, le monopole gaulliste sur le SDECE a disparu et que le SDECE a été l'enjeu d'une lutte avec les autres partis, les réseaux gaullistes ont toujours maintenu un lien privilégié avec ce service. C'est le cas en particulier du service action du SDECE, avec son bras armé, le $11^{\mathrm{e}}$ choc, fondé par Aussaresses. Jacques Foccart était réserviste du $11^{\mathrm{e}}$ choc, et y faisait régulièrement ses périodes. Il a exercé longtemps une forte autorité morale sur cette unité, au point qu'Aussaresses l'a qualifié de « patron hors hiérarchie $»^{16}$. On voit ainsi comment les réseaux gaullistes de résistance engendrèrent les réseaux gaullistes d'après-guerre qui se trouvèrent implantés organiquement au cœur même de l'État. Le mouvement gaulliste, avant d'être considéré comme un parti, doit être appréhendé comme un réseau de réseaux plus ou moins clandestins. Ces réseaux en ont constitué l'ossature, la colonne vertébrale. Dormants pendant certaines périodes, ils pouvaient être réactivés en cas de besoin. En ce sens-là, le parti gaulliste, au même titre que le parti communiste, n'était pas un parti comme les autres. Il existait dans le mouvement gaulliste une véritable culture de l'action clandestine et Jacques Foccart en fut l'incarnation, de même il était au centre du «nexus », de la «toile » permettant de faire communiquer les présidents, l'appareil d'État et le mouvement gaulliste, en s'appuyant à la fois sur les structures, son réseau relationnel et ses réseaux clandestins.

\section{Réseau relationnel et réseaux occultes}

En distinguant ces deux types de réseaux, nous voulons souligner les deux caractéristiques principales qui attirent l'attention lorsqu'on se penche sur le sujet, la dimension relationnelle et la dimension occulte. Bien entendu tout réseau est relationnel. Mais il existe une différence entre les réseaux licites informels qui correspondent au capital relationnel, au carnet d'adresses d'une personne, et les réseaux clandestins, illicites, organisés, qu'ils soient l'émanation de structures publiques ou simplement privés et quasi mafieux. Il n'y a pas de cloison étanche entre les deux types de réseaux, mais plutôt une sorte de continuum. Il est de la nature même des réseaux de permettre l'articulation entre des milieux extrêmement divers, et c'est celui qui se trouve au point 
d'intersection, au "nexus ", au centre de la toile de ces divers milieux qui occupe la position clé. Nous avons vu que c'était le cas de Jacques Foccart.

\section{La dimension relationnelle du réseau Foccart}

26 S'il ne reconnaît pas l'existence de ses réseaux occultes, il s'étend par contre sur son réseau relationnel pour expliquer sa méthode et l'efficacité de son action. Il insiste sur deux types de réseau, ce qu'on peut appeler son réseau personnel de renseignement et son réseau africain.

\section{Le réseau personnel de renseignement}

Il reconnaît qu'il était bien informé, mais il insiste sur le fait qu'il ne s'agit pas d'un réseau illicite, mais d'un réseau de correspondants, c'est-à-dire d'amis, de connaissances bien placés et avec lesquels il entretient des relations (téléphone, visites), ou même d'inconnus qui lui transmettent spontanément des informations à cause de sa réputation :

Philippe Gaillard: Vous déteniez ou receviez des renseignements qui ont dû être très utiles en ces circonstances.

Jacques Foccart: Moins qu'on a pu le dire et pas comme on le croit. Ces gens qui m'informaient n'appartenaient à aucune association, à aucun réseau. Ils me connaissaient plus ou moins ou ils avaient entendu parler de moi, et ils me donnaient des renseignements. Dans des périodes dramatiques comme celles-là, des amis connus et inconnus, des gaullistes ayant appris quelque chose qu'ils pensaient utile de me faire connaître pour la défense du régime, du pays, me téléphonaient, venaient me voir. Je recevais toutes les personnes qui insistaient pour me rencontrer en disant qu'elles avaient une information importante à me communiquer, ce qui n'allait pas toujours sans risque.

[...] Mais je disposais quand même de quelques antennes. En définitive, il est exact que j'étais un homme bien renseigné. Beaucoup de gens sont enclins à penser que quelqu'un qui dispose d'informations nombreuses, précises, exactes et rapides, ne peut les devoir qu'à des moyens louches et à des réseaux clandestins. Pourtant c'était une simple question d'organisation. Pourquoi étais-je bien informé ? Primo, parce que je connaissais beaucoup de personnes, en France et en Afrique, qui étaient elles mêmes bien informées, chacune dans son secteur, en raison de leurs fonctions politiques, administratives ou économiques, et de leurs contacts. Elles connaissaient mes numéros de téléphone et je connaissais les leurs. Nous nous appelions parfois sans motif particulier, pour entretenir nos relations. Secundo, et c'était un effet positif de la réputation qui m'était faite, des inconnus qui voulaient faire connaître une information au gouvernement sans savoir à qui s'adresser pensaient à "appeler Foccart». Tertio, et c'est peut-être la clé de voûte de l'ensemble, j'étais toujours disponible ${ }^{17}$.

Bien entendu, ce réseau personnel de renseignement englobe le réseau africain dont la portée va cependant bien au-delà du renseignement.

\section{Le réseau africain}

Enfin, tout au long de ses témoignages, et nous reviendrons là-dessus, il souligne la fréquence et la profondeur des relations qu'il entretenait avec les dirigeants africains, capital relationnel qui l'a rendu indispensable au général de Gaulle ainsi qu'à Georges Pompidou. Tous les témoignages confirment qu'au cœur de l'action de Jacques Foccart, il y avait ce capital relationnel unique qu'il avait constitué, qu'il s'attachait à entretenir par 
de multiples contacts et des services rendus, et qu'il a mis au service de la politique française. Il a su ainsi transformer les relations d'amitié qu'il avait établies avec les dirigeants africains en véritables ressources politiques. Mais il faut souligner que ce qui avait permis de constituer et de développer ce capital de relations, c'était justement la relation privilégiée de proximité qu'il entretenait avec le général de Gaulle et le président Pompidou. Le secrétariat aux Affaires africaines et malgaches était chargé de l'accueil et, pourrait-on dire, de la prise en charge de toutes les personnalités africaines qui transitaient par Paris. Jacques Foccart recevait les plus importantes d'entre elles à la villa Charlotte, à Luzarches, qui était devenue une sorte d'annexe des résidences officielles. Il était l'hôte attentionné de multiples visiteurs africains qui venaient souvent avec leurs familles. C'est au point qu'il sera décidé, sous Georges Pompidou, conscient de l'importance d'entretenir ses contacts, de faire construire dans sa propriété un pavillon de réception ${ }^{18}$. Pierre Péan insiste sur l'impact de cette hospitalité sur les dirigeants africains francophones :

Ceux-ci ont une confiance absolue en cet homme discret qui les comprend et les connaît. Pour eux, Foccart est non seulement l'accès sûr et direct au Général, mais il est aussi le seul capable de régler tous leurs problèmes, qu'ils soient politiques, personnels ou familiaux. Il n'a qu'un ordre à donner pour que le nécessaire soit fait, pour doter d'une compagne quelque président esseulé, trouver un collège où placer les enfants d'un autre, arranger tel ou tel problème de diplôme concernant la progéniture d'un troisième... Il sait parler une langue qu'ils comprennent... Avec lui, ils ont le sentiment d'être chez eux en France et traités comme des amis. Foccart invite la plupart d'entre eux à la villa Charlotte. Il va aussi les voir à domicile à l'occasion de ses fréquents voyages en Afrique. Il est ou devient l'ami intime de l'Ivoirien Houphouët-Boigny, du Gabonais Bongo, du Sénégalais Senghor, du Camerounais Ahidjo et du Zaïrois Mobutu ${ }^{19}$.

Par là, Jacques Foccart donne l'exemple à l'ensemble de la classe dirigeante française. Le tissu de relations personnelles et d'échanges sociaux au cœur du «réseau francoafricain » est fondé sur les mêmes mécanismes, comme le mentionne Loïk Le FlochPrigent.

Les chefs d'État africains devaient être des « amis » et il existait des relations entre Africains et Français d'Afrique qui maintenaient la stabilité de leur pouvoir. Dans tous les milieux, politique, affaires, sécurité, ces hommes s'entraident de façon naturelle depuis la scolarité des enfants jusqu'aux contrats de développement des routes, des ports, des usines... et du pétrole. Le réseau, c'est le maillage d'intérêts pour l'information et pour la mise en œuvre d'une politique ${ }^{20}$.

31 C'est en grande partie grâce à ces liens d'amitiés systématiquement entretenus que Jacques Foccart a pu exercer l'influence qu'il se reconnaît et qu'on lui reconnaît :

Essentiellement par des contacts directs, nous avions de l'influence sur l'évolution des uns et des autres... Trente ans après, il me reste à titre personnel quelque chose de l'influence que j'avais acquise grâce à la qualité de ces contacts. Je suis convaincu que ces dialogues entretenus sur le plan humain, souvent amical et décontracté, étaient plus efficaces que les meilleures notes d'ambassadeur...21

À lire son Journal, on remarque d'ailleurs qu'il entretenait une relation personnelle de nature différente avec chacun de ses interlocuteurs africains. Parmi ces derniers, deux présidents semblent avoir été privilégies, Houphouët-Boigny et Bongo. Avec HouphouëtBoigny, il entretenait une relation d'amitié profonde et confiante qui n'a fait que s'approfondir avec le temps. Avec Bongo, qui avait été qualifié de " créature de Foccart » et son pays de "Foccartland», en référence aux conditions d'accès au pouvoir d'Omar Bongo et à la place que les réseaux Foccart tenaient dans son pays, les relations semblent 
s'être progressivement distanciées. Au fur et à mesure qu'Omar Bongo s'est transformé en un émir du pétrole, il a pris de l'assurance, et la relation est devenue plus instrumentale ${ }^{22}$. En le lisant, on réalise à quel point la relation qu'entretenait Foccart avec ses interlocuteurs africains demandait du doigté et de la psychologie ; l'art de l'influence est un art délicat.

Mais il ne faut pas que cette insistance justifiée sur les dons relationnels de Jacques Foccart, et sa capacité à se faire des amis en Afrique, cache le revers de la médaille. L'amitié est quelque chose de fort sympathique, mais elle a recouvert en l'occurrence bien des pratiques inavouables de corruption, voire criminelles.

\section{La dimension occulte des réseaux Foccart}

Jacques Foccart a toujours nié l'existence des réseaux Foccart au sens où nous les avons précisés $^{23}$. À lire Jacques Foccart, on a ainsi l'impression que ces réseaux sont purement et simplement le fruit de l'imagination débridée des journalistes et de ses adversaires. Il n'apprécie d'ailleurs pas du tout lorsque le président Georges Pompidou fait à plusieurs reprises et devant témoins, allusion en plaisantant à ses interventions occultes.

Il a, en revanche, reconnu un certain nombre des responsabilités qu'il a exercées auprès d'organismes occultes, publics ou privés, comme le service action du SDECE et son $11^{\mathrm{e}}$ choc, ses fonctions auprès du général de Gaulle concernant les questions de renseignement et de sécurité, et enfin ses responsabilités au sein du SAC, sur lesquelles nous reviendrons. Il s'est constamment défendu d'être à l'origine des mauvais coups qu'on lui a prêtés, son argument, peu convaincant mais efficace auprès des gaullistes étant de dire :

Je suis resté pendant vingt-trois ans à côté du général de Gaulle qui n'était pas un naiff et qui connaissait bien le comportement de ses collaborateurs; il n'aurait certes pas supporté vingt-quatre heures quelqu'un qui aurait préparé des mauvais coups $^{24}$.

Plus loin, il nous dit :

Jacques Foccart : J'ai d'excellents correspondants... ce qu'on a appelé mes réseaux. Philippe Gaillard : Correspondants, oui, réseaux, non. Où est la différence ?

Jacques Foccart : Je vous ai dit que je connaissais beaucoup de gens bien informés. Je leur téléphonais, ils me téléphonaient. Un réseau est un ensemble de correspondants organisés, hiérarchisés, souvent rémunérés. Je n'ai jamais eu à ma disposition ni réseau ni rien qui y ressemble ${ }^{25}$.

Quand on sait comment se comporte le genre de service avec lequel il entretenait des relations aussi étroites et officielles, il est difficile de prétendre qu'on est hors du coup. Ces services entrent bien dans la catégorie des réseaux tels qu'il les définit lui-même. Ce côté sombre du personnage, systématiquement nié et pour cause, a été pourtant reconnu par certains de ses partisans. Ainsi dans sa thèse, Robert Bourgi, le fils d'un vieil ami libano-sénégalais de Jacques Foccart, écrit en faisant allusion aux débuts de Jacques Foccart en Afrique, qu'il recourt

[...] à divers stratagèmes propres aux organisations et sociétés secrètes : formation de réseaux de renseignement, enquêtes sur les opinions politiques des administrateurs et fonctionnaires coloniaux, tentative de noyautage des milieux d'affaires installés en Afrique ${ }^{26}$.

Ces réseaux gaullistes sont ainsi implantés en Afrique dans les milieux français bien avant le retour au pouvoir du général de Gaulle. Jacques Foccart s'appuie d'abord sur les gaullistes, anciens de la France libre. Il pénètre ensuite les milieux d'affaires. Lui-même 
développe son entreprise d'import-export, la Safiex, au-delà des Antilles, ce qui fait dire à Pierre Péan :

Il ne quadrille pas seulement l'Afrique politiquement mais commercialement ${ }^{27}$.

Son ami Maurice Robert met en place le SDECE-Afrique à partir de Dakar, avec un réseau de postes de renseignement et de liaison (les PRL), chargé de suivre l'évolution politique. Il s'agit, selon Jacques Foccart lui-même, de la partie quasi officielle, le SDECE ayant aussi son organisation clandestine ${ }^{28}$ dans chaque pays. Il y a ainsi un colonel français en poste auprès de chaque président ami. Ce service fera appel aussi à des mercenaires dont Bob Denard est le plus connu, et qui a été impliqué dans une attaque contre le Bénin et aux Comores à diverses reprises. Jacques Foccart a nié avoir entretenu des rapports avec lui. $C^{\prime}$ est sans doute jouer sur les mots, car son officier traitant était, semble-t-il, Maurice Robert. Ce dernier a témoigné au procès de Bob Denard qu'il était un patriote et avait rendu de grands services à la France ; il a reconnu implicitement les liens qu'il entretenait avec ses services. L'expression de «corsaire de la République» que revendiquait Bob Denard, exprime bien l'ambivalence de son statut de "faux-vrai mercenaire", à distinguer des «vrais-faux mercenaires» que sont les membres des services qui se font passer pour mercenaires.

Les services français apportaient de plus leur assistance technique aux services spéciaux locaux, comme, par exemple, au Cameroun, à la sinistre Dirdoc de Fochivé.

Enfin, les gardes présidentielles des présidents africains sont truffées d'anciens militaires ou d'anciens des services, plus ou moins en rapport avec leur institution d'origine, et même de membres du SAC. Ainsi Debizet, qui avait quitté la direction du SAC en 1960 à cause de l'Algérie, redeviendra secrétaire général en 1968 : il était responsable de la garde présidentielle d'Omar Bongo au moment du massacre d'Auriol.

9 Pour couronner le tout, il faut enfin souligner le rôle d'Elf sur lequel on est mieux renseigné aujourd'hui, à la suite des scandales multiples qui l'ont atteint. Loïk Le FlochPrigent, ancien directeur d'Elf, nommé par François Mitterrand, a clairement décrit ce qu'il appelle le «système Elf» et son témoignage confirme tout ce qu'on pouvait subodorer. Il a rappelé que c'est de Gaulle qui a créé Elf :

Le problème du général de Gaulle était d'abord d'assurer l'indépendance énergétique de la France. Pour cela, il crée une grande société pétrolière qu'il confie au corps des Mines et à son plus éminent représentant, Pierre Guillaumat. Les hommes qui vont diriger Elf sont non seulement des ingénieurs, des spécialistes des questions nucléaires et pétrolières mais également des gaullistes fidèles... De Gaulle veut une société complètement contrôlée par l'État, qui soit son bras séculier dans le pétrole et pour affirmer sa politique africaine. Elf n'est pas seulement une société pétrolière, c'est une diplomatie parallèle destinée à garder le contrôle sur un certain nombre d'États africains, surtout au moment clé de la décolonisation... Et c'est justement parce que cette société avait un objet politique et diplomatique en Afrique qu'elle a de tous temps financé les services secrets... parallèlement à l'exploration et à l'exploitation du pétrole, un certain nombre d'opérations opaques étaient organisées afin de préserver la stabilité politique dans certains pays. Les méthodes étaient opaques, mais le système a été créé pour permettre cette opacité ${ }^{29}$

Il poursuit en mentionnant le rôle d'Elf dans le financement des partis gaullistes et dans la relation avec les chefs d'État africains. Concernant les fameux réseaux, il souligne le rôle historique de Jacques Foccart ${ }^{30}$, qu'il qualifie de "personnage central » des réseaux gaullistes. Il distingue, au sein de la société, trois grandes familles de réseaux : le réseau politique, le corps des Mines et le réseau du renseignement ${ }^{31}$. Elf avait ses propres 
services de sécurité en liaison étroite avec le SDECE. Ainsi, Maurice Robert, le fondateur $\mathrm{du}$ SDECE-Afrique, qui fut ambassadeur au Gabon, quitta ce poste pour prendre la direction des services de sécurité d'Elf. L'osmose était ainsi totale entre le réseau Foccart, le mouvement gaulliste, le SDECE, Elf et les dirigeants africains. On peut dire que la Françafrique reposait sur deux piliers qui se recoupaient et se confortaient mutuellement, le réseau Foccart et Elf. Les seuls réseaux qui ne sont jamais mentionnés durant la période Foccart sont les réseaux franc-maçons, alors que le Grand Orient a été beaucoup cité au début des années quatre-vingt-dix, et la Grande Loge nationale de France (GLNF) se trouve toujours sous les feux de l'actualité.

Pour synthétiser et conclure tout ce qui précède, nous citerons, malgré sa longueur, ce texte de Daniel Bach qui a le mérite de souligner l'essentiel :

[...] In both France and Francophone Africa, the Franco-African networks associated with Jacques Foccart... sustained a powerful and hidden nexus of influence which permeated state and politics until the mid seventies. The "Foccart networks ", as they were commonly refered to, relied on French state institutions and the Gaullist party to build up of a web of connexion along these and within the military, state owned corporations, private companies, secret services, private soldiers and, not the least, ruling elites in Africa. The result was an extraordianry web of influence wich, in France, succeeded in establishing its influence over state policy formation when Africa was at stake, with the result that institutions such as the National Assembly or even France's foreign affairs ministry was confined into subsidiary or purely formal functions. Across the continent, the so-called Foccart networks were able to cast a long and pervasive shadow behind the politics of most of the newly independant francophone states: international patronage superseded inter-state relations as a modus vivendi against a background profligate with the diversion of contract allocations procedures, extensive appeal to tied aid, systematic overinvoicing, the use of ODA disbursment for the purpose of party funding, and the unrestrained availability of the Franc zone financial system to launder the proceeds of illicit enrichment and corruption. Political stability and the preservation of the status quo were key to the prosperity of such a politico-financial and criminal nexus : attempts to seccede from the Francophone pack or threaten its cohesion were accordingly kept under close scrutiny through the manipulation of policy outcomes by the network of advisors and experts directly connected to both the French and African presidents and ministers. If necessary, force was used, overtly so as to confort the power of friendly heads of states, as occured in Gabon (1964), or Chad (1968), but also covertly, in order to neutralise domestic opposition to friendly regimes, and destabilize those considered unfriendly as Sekou Touré's Guinea, or dangerous as Lumumba's Congo or Nigeria during the Nigerian civil war ${ }^{32}$.

Les leçons de ces pratiques et de cette politique ont été fort bien tirées par Pierre Marion, l'ancien directeur-fondateur de la DGSE :

[L'entreprise Foccart] s'est appliquée, grâce à l'appui puissant du général de Gaulle et à la force de son prestige, à perpétuer en Afrique une situation néo-coloniale... Elle a contribué à l'installation de régimes autoritaires et au développement d'un système généralisé de corruption. Les réseaux Foccart ont eu deux autres effets pervers. D'abord, leurs actions mélangent à un haut degré influence politique et commerce, pour ne pas dire affairisme. On ne sait pas très bien où s'arrête l'intérêt général et où commencent les intérêts privés. Ensuite les inversions de sens se produisent dans l'influence, la France devenant l'objet de manipulations, soit par des personnes ou des intérêts liés à ces réseaux, soit par des fonds occultes provenant de caisses noires de certains chefs d'État africains ou moyen-orientaux, au premier rang desquels le Gabon et l'Irak. Les financements des partis politiques et des campagnes électorales en sont les principaux bénéficiaires ${ }^{33}$. 
question qu'on peut poser en conclusion, c'est de savoir si ces objectifs pouvaient être atteints par d'autres méthodes? On ne peut refaire l'histoire, mais la réponse est non. La nature même des objectifs impliquait les méthodes qui ont été utilisées, d'autant plus que sur les objectifs de grande politique se greffaient d'autres objectifs encore plus douteux. Était-il envisageable alors de mener une politique préservant certains intérêts légitimes de la France et le respect d'une véritable indépendance des États africains? C'est malheureusement improbable dans le contexte politique de l'époque, mais il n'est pas interdit de rêver.

\section{NOTES}

1. Stephen Smith et Antoine Glaser, Ces Messieurs Afrique, tome I, Le Paris-Village du continent noir, Paris, Calmann-Lévy, 1992, 236 p., p. VIII.

2. François-Xavier Verschave, La Françafrique, Paris, Stock, 1998, 380 p. ; id, Noir Silence, Paris, Les Arènes, 2000, 597 p. et Noir Chirac, Paris, Les Arènes, 307 p., 2002.

3. Jean-Pierre Dozon a parlé au cours de ce colloque d'un État franco-africain. Il s'agit de la même idée, sauf que la Françafrique s'étend au-delà de l'État.

4. Jean-François Médard, "Les avatars du messianisme français en Afrique ", Afrique politique, Paris, Karthala, 1999, p. 17-34.

5. Jean-François Médard, "France-Afrique : within the family ", in Donatella Dellaporta et Yves Mény (eds.), Democracy and Corruption in Europe, London and Washington, Pinter, 1997, 208 p., p. 22-34.

6. Jean-François Médard, "L'Afrique et la corruption internationale", Revue internationale de politique comparée, vol. IV, $\mathrm{n}^{\circ} 2,1997$, p. 413-440.

7. Pierre Biarnès, Les Français en Afrique noire de Richelieu à Mitterrand, Paris, Armand Colin, 1987, 447 p., cité dans Foccart parle, I, p. 218.

8. Ibid.

9. Foccart parle, I, p. 213.

10. Ibid., p. 226.

11. Foccart parle, I, p. 149.

12. Sur les rapports entre la France, le Nigeria et la Côte d'Ivoire, voir Daniel Bach, « Le général de Gaulle et la guerre civile au Nigeria, Centre d'étude d'Afrique noire / Institut Charles-deGaulle, La Politique africaine du général de Gaulle, 1968-1969, Paris, Éditions Pédone, 1980, 421 p.; «L'insertion ivoirienne dans les rapports internationaux», André Fauré et Jean-François Médard, (dir.), État et bourgeoisie en Côte d'Ivoire, Paris, Karthala, 1982, 270 p., p. 89-121.

13. Foccart parle, I, p. 183.

14. Pierre Péan, L'Homme de l'ombre, Paris, Fayard, 1990, 585 p., p. 420.

15. Foccart parle, II, p. 19.

16. Ibid.

17. Foccart parle, I, p. 354-355.

18. Ibid., II, p. 87.

19. Pierre Péan, op. cit., p. 294.

20. Loïk Le Floch-Prigent, Affaire Elf, affaire d'État, Paris, Le Cherche-midi éditeur, 2001, 254 p., p. 78. 
21. Foccart parle, I, p. 218.

22. Omar Bongo, Blanc comme nègre, Paris, Grasset, 2001, 277 p., p. 181-182.

23. On retrouve la même dénégation plus tard à propos des réseaux, lorsque l'ancien collaborateur de Jacques Chirac et ancien ministre de la Coopération nous dit: « et quand on parle des réseaux Pasqua, Roussin, Foccart... Je crois qu'en fait ce sont des liens d'amitié qui existent entre les uns et les autres. Ce qui compte c'est que nos amis viennent nous voir lorsqu'ils ont des problèmes ".

24. Centre d'Étude d'Afrique Noire/Institut Charles-de-Gaulle, op. cit., p. 275.

25. Foccart parle. II, p. 160.

26. Cité par François-Xavier Verschave, La Françafrique, op. cit., p. 99.

27. Pierre Péan, op. cit., p. 192.

28. Foccart parle, I, p. 183.

29. Loïk Le Floch-Prigent, op. cit., p. 53-52.

30. Ibid., p. 77.

31. Ibid., p. 76.

32. Daniel Bach, International Relations Through the African Looking Glass, à paraître.

33. Pierre Marion, La Mission impossible, à la tête des services secrets, Paris, Calmann-Lévy, 1991, 259 p., p. 106. 